agency is committed to improving diagnostic testing," Sternthal said.

Meanwhile, PHAC recommends that doctors diagnose on the basis of clinical assessment, rather than relying on lab tests. Once patients have early symptoms, PHAC recommends 14 to 21 days of antibiotic treatment. However, PHAC suggests that postexposure antibiotic therapy be considered in known endemic areas.
Dr. Allen Steere, the Massachusetts physician who published the first conclusive account of Lyme disease in 1977, says not all patients present with the classic bull's eye-shaped skin rash that many doctors look for to diagnose Lyme. Steere, an infectious disease specialist at the Center for Immunology and Inflammatory Diseases at Massachusetts General Hospital, believes that chronic Lyme disease exists and can result from misdiagnosis as another type of illness or lack of treatment initially.

The best advice for people is to prevent tick bites by avoiding tickinfested areas, using repellant, tucking pants into socks and wearing closed-toed shoes, and conducting daily tick checks. — Laura Eggertson, Ottawa, Ont.

CMAJ 2014. DOI:10.1503/cmaj.109-4850

\title{
Portal simplifies medical registration
}

$\mathrm{T}$

The Medical Council of Canada (MCC) is rolling out a national Web portal to simplify medical assessment, credentialing and registration.

Using physiciansapply.ca, medical graduates and practising physicians can register for MCC examinations, digitally share exam results and other credentials stored in the council's central repository, and request verification and translation of international credentials.

Once the system is fully deployed, users will also be able to apply for a medical licence to any of Canada's 13 provincial and territorial medical regulatory authorities through a single electronic process.

"Currently and in the past, candidates have had to apply to each province and territory individually," says MCC Executive Director Dr. Ian Bowmer. "If you wanted to apply to more than one province, you'd have to navigate the same process a number of times."

That system created unnecessary barriers for foreign doctors, "and even our own Canadian graduates, who often want to practise in more than one province early in their careers," Bowmer says.

With the new portal, "our goal was to have a one-stop application process for international medical graduates, because they needed more support than Canadian applicants," he says. "But what we've got is a system that's simpler for everyone."

In phase one of the rollout, the portal was opened to new candidates in Alberta and Nova Scotia, including Canadian medical residents, practising physicians relocating from other provinces and

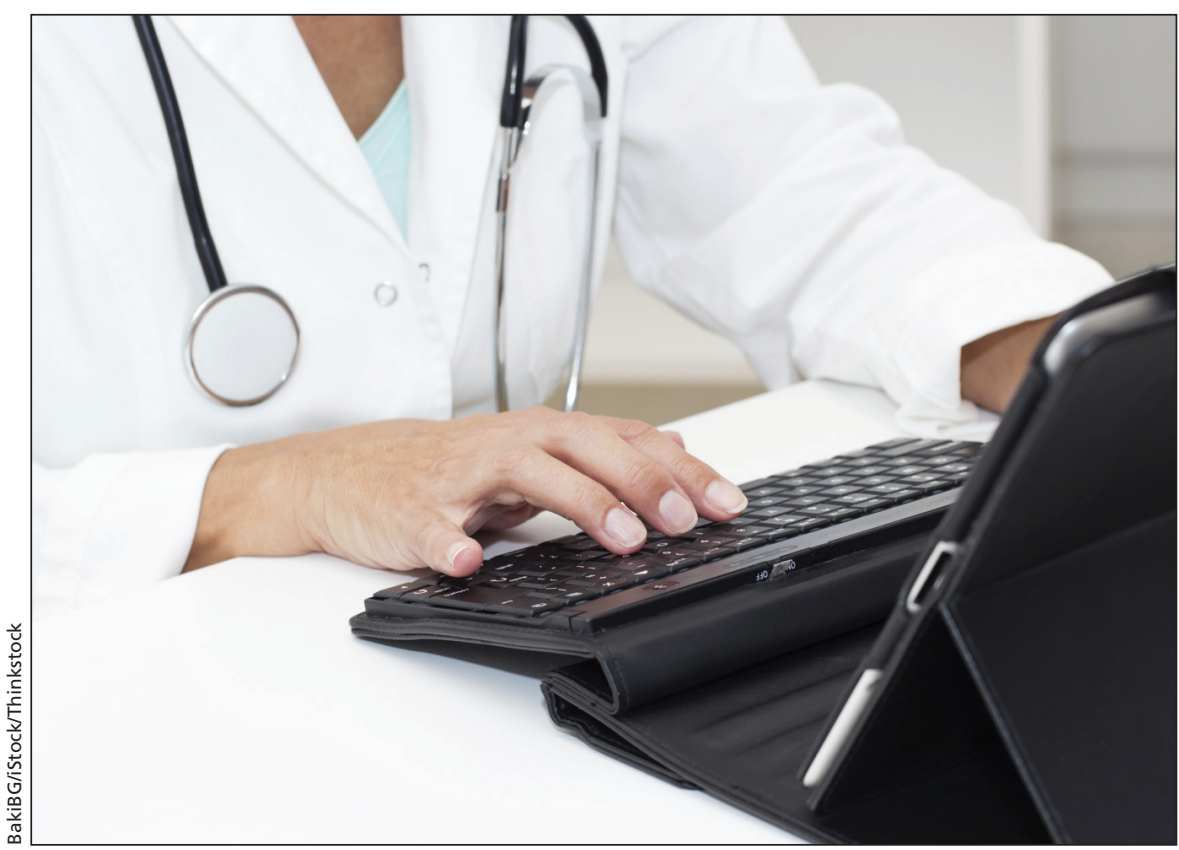

A new Web portal will let medical graduates apply for registration in multiple Canadian jurisdictions using a standardized form.

international medical graduates. Other medical regulatory authorities across Canada will adopt the new system over the next two years, says Bowmer.

Users will pay a one-time fee - $\$ 260$ in 2014 — for lifetime access to the portal. The system will also save user data to automatically populate new applications with previously entered details.

Transitioning to the new portal has been "fairly seamless," says Dr. Kate Reed, assistant registrar for the College of Physicians and Surgeons of Alberta. It helped that the province already had a fully electronic application process for medical registration, she notes.

According to Dr. William Lowe, deputy registrar for the College of Physi- cians and Surgeons of Nova Scotia, smaller jurisdictions with paper-based processes may be in for a bumpier ride. However, they may also have the most to gain from the new streamlined system.

"If it's easy to apply to five or six jurisdictions at once, you may be more likely to apply to Nova Scotia," he says. "I believe we're likely to see more applications than we would have previously.'

Although the portal will standardize the process of applying for medical registration, jurisdictions will still assess applications according to their own criteria. - Lauren Vogel, CMAJ

CMAJ 2014. DOI:10.1503/cmaj.109-4838 